
\title{
Reseracth Sulure \\ Three-Dimensional Deep Learning to Automatically Generate Cranial Implant Geometry
}

\section{Yau-Zen Chang ( $\boldsymbol{\nabla}$ zen@cgu.edu.tw)}

Chang Gung University

\section{Chieh-Tsai Wu}

Chang Gung Memorial Hospital

\section{Yao-Hung Yang}

Chang Gung University

\section{Research Article}

Keywords: 3D, framework, model, geometry

Posted Date: January 18th, 2021

DOI: https://doi.org/10.21203/rs.3.rs-139772/v1

License: (c) (i) This work is licensed under a Creative Commons Attribution 4.0 International License.

Read Full License 


\title{
Three-Dimensional deep learning to automatically generate cranial implant geometry
}

\author{
Chieh-Tsai $\mathrm{Wu}^{1}$, Yao-Hung Yang ${ }^{2} \&$ Yau-Zen Chang ${ }^{1,2^{*}}$ \\ ${ }^{1}$ Department of Neurosurgery, Chang Gung Memorial Hospital, Taoyuan 33305, \\ Taiwan. ${ }^{2}$ Department of Mechanical Engineering, Chang Gung University, Taoyuan \\ 33302, Taiwan. *Correspondence and requests for materials should be addressed \\ to Y.Z.C. (email: zen@cgu.edu.tw)
}

We present a 3D deep learning framework that can generate a complete cranial model using a defective one. The Boolean subtraction between these two models automatically generates the geometry of the implant required for surgical reconstruction. There is little or no need for postprocessing to eliminate noise in the implant model generated by the proposed approach. The framework can be used to meet the repair needs of cranial imperfections caused by trauma, congenital defects, plastic surgery, or tumor resection. Traditional implant design methods for skull reconstruction rely on the mirror operation. However, these approaches have great limitations when the defect crosses the plane of symmetry or the patient's skull is asymmetrical. The proposed deep learning framework is based on an enhanced three-dimensional autoencoder. Each training sample for the framework is a pair consisting of a cranial model converted from CT images and a corresponding model with simulated defects on it. Our approach can learn the spatial distribution of the upper part of normal cranial bones and use flawed cranial data to predict its complete geometry. Empirical research on simulated defects and actual clinical applications shows that our framework can meet most of the requirements of cranioplasty.

Cranioplasty ${ }^{1,2}$ is a surgical procedure in which cranial implants, or prostheses, are used to repair skull defects caused by trauma, congenital defects, plastic surgery, or tumor resection. The cranial implants must have an appropriate convex shape and fit accurately to the boundary of the defect. Their design usually involves time-consuming human-computer interaction using specific software and requires expertise in the medical field. For instance, Chen et al. ${ }^{3}$ utilized the geometry information of the mirrored model as the base to generate the implant model.

Considering that cranial defects may cross the plane of symmetry, and human cranial bones are usually asymmetrical, it is impractical to use the mirroring operation 
to generate the implant geometry. Therefore, there is a great need for automatic design of cranial implants.

In recent years, there have been substantial progresses in the image inpainting technology based on deep learning ${ }^{4}$. Image inpainting is the process of completing or repairing missing areas in a two-dimensional image. For example, Yan et al. ${ }^{5}$ introduced a shift-connection layer to the U-Net architecture ${ }^{6}$ for image completion that exhibits fast speed with promising fine details. Liao et al. ${ }^{7}$ proposed a deep convolutional neural networks scheme to explicitly separate content and style that generates fine-detailed and perceptually realistic inpainting results for structural and natural images. Besides, Pathak et al. ${ }^{8}$ combined the autoencoder network model ${ }^{9,10,11}$ with Generative Adversarial Network ${ }^{12}$ (GAN) to repair images and found that, in addition to reconstruction loss, an adversarial loss is beneficial in producing clear results. The schemes of Iizuka et al. ${ }^{13}$, Wang et al. ${ }^{14}$ and Jiang et al. ${ }^{15}$ are all based on the combination of autoencoder model ${ }^{9,10,11}$ and $\mathrm{GAN}^{12}$, in which a global context identifier and a local context identifier are used.

Compared with 2D images, 3D geometric models require more computing power to process. In the inpainting of 3D models, the neural network architecture of Han et al. ${ }^{16}$ is divided into two parts, where the "Global Structure Inference" is responsible for the restoration of $32 \times 32 \times 32$ low-resolution data, and the "Local Structure Refinement” part is responsible for refinement. Wang et al. ${ }^{17}$ also used GAN ${ }^{12}$ to train an Encoder-Decoder network ${ }^{9,10,11}$ to repair defects in 3D images with a resolution of $32 \times 32 \times 32$ voxels. Dai et al. ${ }^{18}$ used a 3D encoder-predictor network to repair the defects of $3 \mathrm{D}$ images with a volumetric resolution of $32 \times 32 \times 32$. The images are then replaced by higher resolution data by direct search. In addition, Wang et al. ${ }^{19}$ proposed a scheme that contains a local GAN ${ }^{12}$ and a global $\mathrm{GAN}^{12}$ to repair 3D mesh model in $80 \times 80 \times 80$ voxels. The performance demonstrations of these contributions, however, are all based on simple geometric shapes such as airplanes, desks, and chairs.

Recently, Morais et al. ${ }^{20}$ proposed a deep learning approach, called Volumetric Convolutional Denoising Autoencoder, to perform 3D shape completion on defected skull models. This approach was evaluated on a full-skull reconstruction task and no verification of the generated implant geometry was provided. The deep learning approach of $\mathrm{Li}$, J. et al. ${ }^{21}$ is carried out in two steps using two neural networks. First, a network is trained to reconstruct the low-resolution version to locate the defective area. Second, another neural network is trained to make detailed implant predictions.

In addition, Shi \& $\mathrm{Chen}^{22}$ proposed a convolutional neural network of the autoencoder ${ }^{9,10,11}$ structure with an auxiliary path to predict the 3D implant from inpainting 2D slices of different axes. Matzkin et al. ${ }^{23}$ used a 3D version of the standard U-Net architecture ${ }^{6}$ to compare two different approaches: direct estimation of the implant, and the reconstruct-and-subtract strategy, where the complete skull is first reconstructed, and then the defective model is subtracted from it to generate the implant. Before training, all the images were registered to an atlas space which is constructed by averaging several healthy head CT images. They concluded that the latter tends to generate noise in the implant models. In the succeeding work of Matzkin et al. ${ }^{24}$, an approximate shape prior, which is constructed by averaging several healthy head CT images, is concatenated with the input model to provide supplementary context information to the network. This modification is reported to facilitate the robustness of the model for out-of-distribution cases. 
Nevertheless, these skull repair techniques are limited in feasible resolution, and the defects are all regular shapes produced by spherical or cubic masks. These shortcomings reduce its applicability in clinical practice.

The purpose of this research is to develop practical 3D inpainting techniques to automatically generate the geometry of the cranial implant, thereby eliminating subjectivity. As shown in Fig. 1, the proposed cranioplasty procedure begins with integrating a defective 3D skull model using a CT-scanned image dataset. A completed cranial model is then automatically created by the proposed deep learning system. To reduce the computational burden, this study only generates the upper part of the cranium. After that, an implant model is obtained by subtracting the original defective model from the completed model. Subsequently, 3D printing technology is used to make a template, and the molding procedure is applied to create the implant, which is made of bone cement here, required for the repair surgery.

In the casting and molding process to create the implant, silicone rubber was used to make the mold to capture geometric details. We had chosen bone cement to make hand-crafted skull patches for more than 16 years and found the material satisfactory. Other biocompatible materials ${ }^{1}$ can also be molded to match the shape of the defect in the same way.

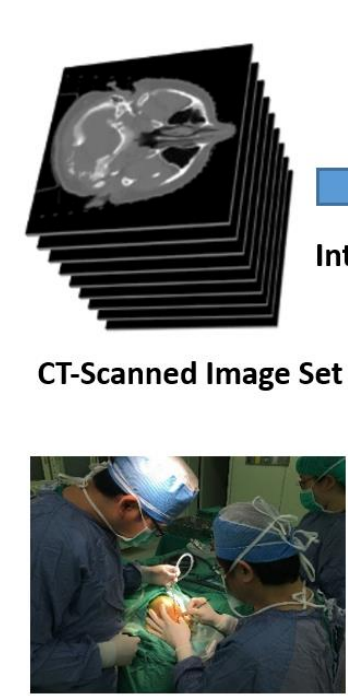

Repair Surgery

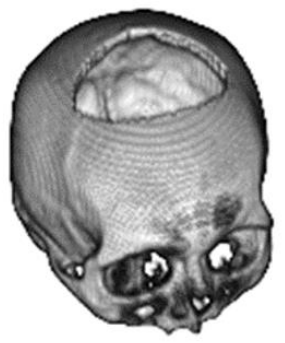

Original 3D Model

The Proposed 3D Deep

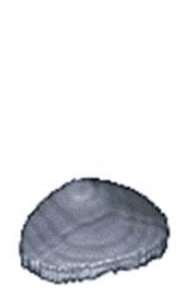

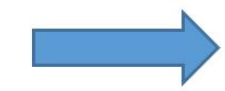

Learning System

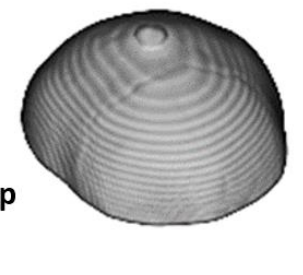

Completed 3D Model

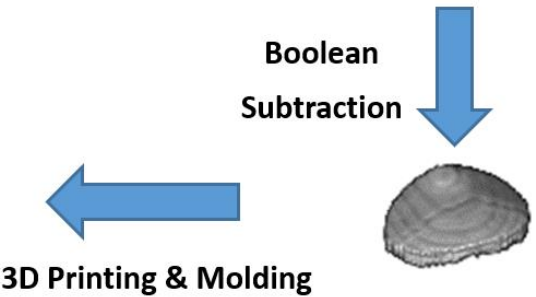

Implant 3D Model

Figure 1. Cranial reconstruction surgery flowchart. 


\section{Results}

Numerical Study. This section uses simulation cases to describe the quantitative performance of the proposed framework. Simulation is needed because in real situations it is impossible to obtain a perfect (ground-truth) implant model from a defective skull.

Figure 2 demonstrates 4 inpainting cases. The upper parts of the defective skulls are displayed in the top view and isometric view in the first and third rows, respectively. The second and fourth rows present the complete skulls generated by the proposed system. The ideal (ground-truth) implants and the created implants are shown in the fifth and sixth rows, respectively.

In the numerical evaluation, we created regular and irregular holes on intact 3D cranium models. The difference between cylindrical and ellipsoidal defects lies in the boundary of the defects. The former is parallel to the axial direction, while the latter is curved, as shown in the flawed skulls of Fig. 2. Please note that all defects pass through the central plane in these cases and therefore cannot be created based on the traditional symmetry assumption.

The implants in the sixth row are obtained by subtracting the original flaw skull models from the generated complete models. If a generated implant is denoted as $P^{*}$ and its corresponding ideal one is expressed as $P$, the volumetric error rate, denoted as $r$, is defined as

$$
r=\frac{\left\|P-P^{*}\right\|_{1}}{\|P\|_{1}} \cdot 100 \%
$$

where the 1-norm is used. The last row of Fig. 2 quantitatively summarizes the repair performance of the proposed scheme. We can find that the proposed deep learning system achieves a volumetric error rate of less than $8.2 \%$ in this case study.

In addition, to understand the limitations of the repair ability of the proposed scheme, we created defects of various sizes and positions on the skull model for numerical study. According to the numerical investigation, detailed in the Supplementary Material, the system can produce satisfactory implants for defects up to $35 \%$ in volume.

Surgical Implementation. The proposed deep learning system has been used in implant generation for clinical applications. This section describes one of these successful implementations.

A 12-year-old boy with a congenital craniofacial defect sought surgical treatment. Computed tomography showed that the longest crack in his sagittal suture was $124 \mathrm{~mm}$ in diameter. As shown in Fig. 3, the proposed deep learning system generated an adequate $3 \mathrm{D}$ geometry of the implant required to repair the defect.

It is worth mentioning that although the system has been trained on simplified cylindrical and elliptical defects, the geometry of the generated implant is satisfactory for actual implants with irregular boundaries. 
Inpainting Defective Cranial Models by the Proposed Scheme

\begin{tabular}{c|c|c|c|}
\hline Defect Type & Cylinder \\
Defective \\
Skull \\
(Top View)
\end{tabular}

Figure 2. Automatic generation of implants for defective cranial models by the proposed deep learning system. The defects are made by Boolean subtraction of 4 types of 3D masks from an intact skull model. 


\section{Surgical Treatment of a Cranial Defect}

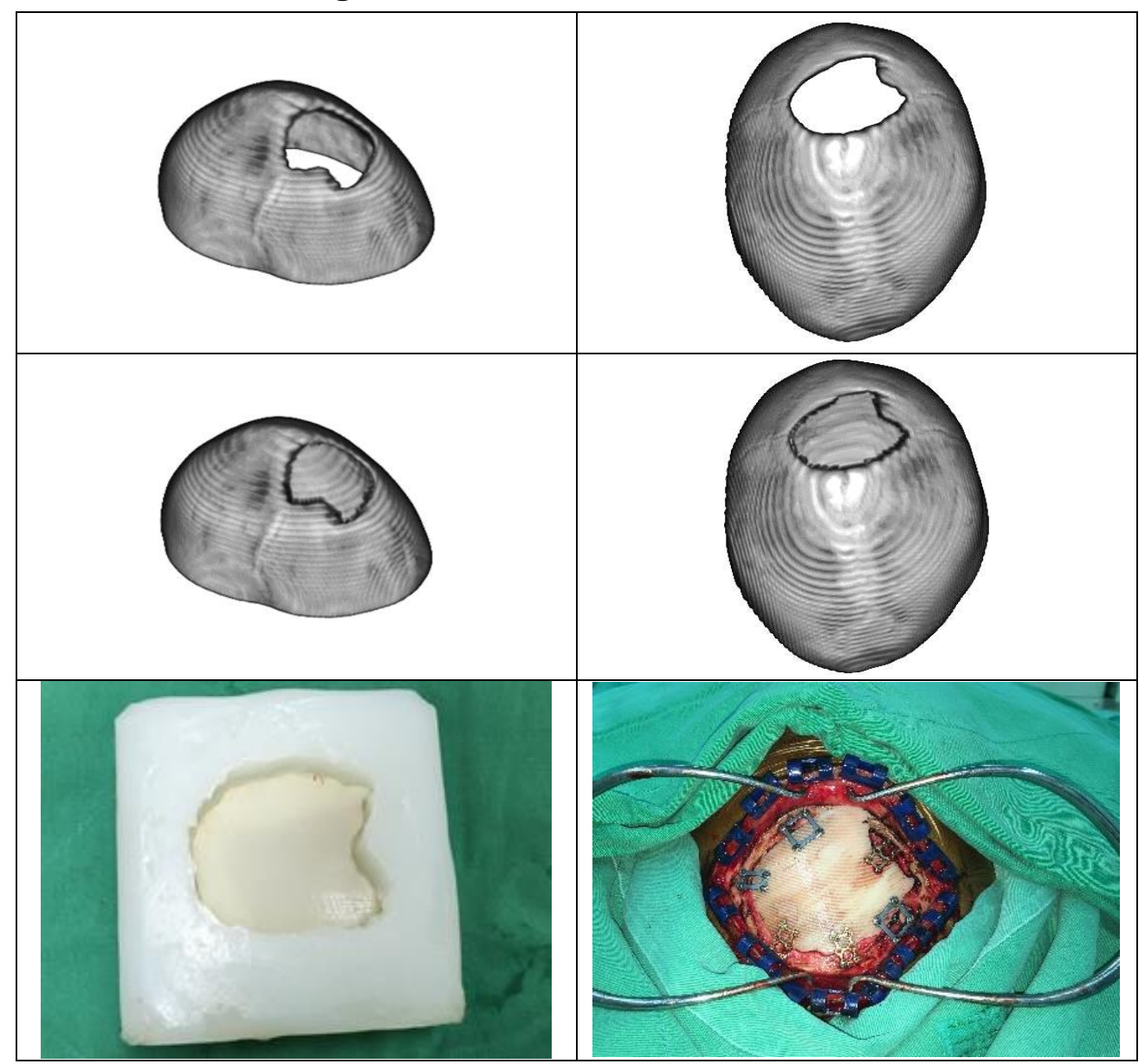

Figure 3. Clinical cranial reconstruction. The first row shows the isometric and top views of the original cranial model. The second row shows the isometric and top views of the repaired cranial model using the implant created by the proposed deep learning system. The last row shows the bone-cement implant molded with silicone rubber (left photo), and a scene of the surgery when the implant was fixed to the skull (right photo).

\section{Discussion}

We propose a 3D deep learning framework to repair defective cranial models. The performance of the framework was investigated in both simulated and clinical cases to verify its applicability.

The capability of the network is made possible through its architecture and training methods. For training, the network inputs are defective 3D models, and the target outputs are the corresponding intact models. This end-to-end learning scheme is essential to effectively train this high-resolution $3 \mathrm{D}$ problem because the training is based on supervised learning instead of relying on indirect information, such as the feedback signal provided by the discriminator in the adversarial networks.

Besides, the Graphics Processing Unit (GPU) can effectively improve the computational efficiency of deep learning. The network is created with as concise 
architecture as possible to alleviate computational requirements. The proposed network only requires a graphics card-enhanced desktop PC to compute, which makes the system a vast potential in many clinical applications.

There are several limitations of the proposed approach, however. First, 7,154 sets of skull models for the deep learning system training were created through the data augmentation technology from 73 skull models. Although case studies strongly support this approach, further clinical trials are needed to evaluate its feasibility for diverse patients. Second, due to the shortage of computing resources, the repairable area is limited to the upper part of the cranium.

Regarding these limitations, this study is a preliminary work, and we believe that it can provide incentive for future advanced research. We plan to expand the scheme to include more areas in the cranial model for reconstruction, such as the area in the zygomatic bones and temporal bones.

\section{Methods}

Cranial Dataset. The dataset used for this study is the DICOM (Digital Imaging and Communications in Medicine) metadata collected in the Department of Neurosurgery, Chang Gung Memorial Hospital, Taoyuan, Taiwan. Being authorized by the Institutional Review Board with IRB No. 201900991B0 and Clinical trial/research Consent No. 201801697B0C601, any protected health information was removed from the DICOM metadata.

Each computed tomography (CT) image is with a resolution of $512 \times 512$, but the interval between the images can be $0.3 \mathrm{~mm}, 0.435 \mathrm{~mm}, 0.5 \mathrm{~mm}, 0.8 \mathrm{~mm}, 1.0 \mathrm{~mm}, 1.25$ $\mathrm{mm}$, or $3.0 \mathrm{~mm}$. CT data contains bones and other tissues, and each patient's taking conditions are different. We set the intensity threshold in the interval of $[1200,1817]$ according to the Hounsfield unit ${ }^{25}$ to preserve the bone tissue in the data.

Also, the number of parameters in the network is proportional to the complexity of the inpainting task. Therefore, enough examples, at least thousands of data sets, are needed to train the network. Unfortunately, after sifting through 327 sets of collected data, only 73 sets are usable, because many of them are incomplete or applied with bone screws. Hence, we rotate, tilt, and vertically translate the 3D medical images, resulting in $73 \times 7 \times 7 \times 2=7,154$ sets of augmented data ${ }^{26}$. The operations are with intervals of 2 degrees for the rotation and tilting, each with 7 alternatives, and 2 voxels for translation.

Due to calculation efficiency considerations, down-sampling is usually required. The original resolution of all collected DICOM metadata on the XY plane is $512 \times 512$ pixels. After weighing the conflict between modeling quality and calculation requirements, we have that at least a $112 \times 112$ plane resolution should be maintained. To further alleviate the computational burden, we only cropped the upper part of the skull models, resulting in normalized datasets with a volumetric resolution of $112 \times 112 \times 40$. 
The Proposed 3D Deep Learning Network. Although 2D image completion technology has made significant progress recently, 3D shape processing involves higher dimensions and is still very challenging. Considering that the human skulls have a similar topology, we manage the system to be trained through end-to-end supervised learning.

The inpainting system is based on a 12-layer deep learning network. In each pair of training sample, the input to the network is a flawed 3D cranial model with a volumetric resolution of $112 \times 112 \times 40$, and the output is the corresponding intact model. Table 1 and Fig. 4 give an overview of its architecture.

The architecture begins with an 8-channel convolution layer and follows with an encoder-decoder structure. The encoder part contains three 3D convolution layers, which are equipped with the Rectified Linear Unit (ReLU), and each is succeeded with a maximum pooling (or max pooling) layer. This part reduces the data size initially to the bottleneck, also known as latent space.

There are four 3D dilated convolutional layers after the encoder part, which are equipped with the ReLU activation function. These mid-layers are important for collecting more structural information surrounding the missing parts. Afterwards, the output of the convolution layers is expanded to higher resolution using 3 deconvolution layers consisting of convolutional and up-sampling layers. Finally, the output layer is a convolutional layer with a sigmoid function to normalize the output to the $[0,1]$ range.

Moreover, we add skip-connection in the network between the corresponding encoder and decoder layers, and between the neighboring mid-layers. The arrangement helps to enhance the prediction ability of the decoding process and prevent the gradient vanishing in the deep neural network. The architecture forms a network with a total of 8,269 trainable parameters. This concise neural network model is realized by reducing the number of channels of the kernel to its performance limit.

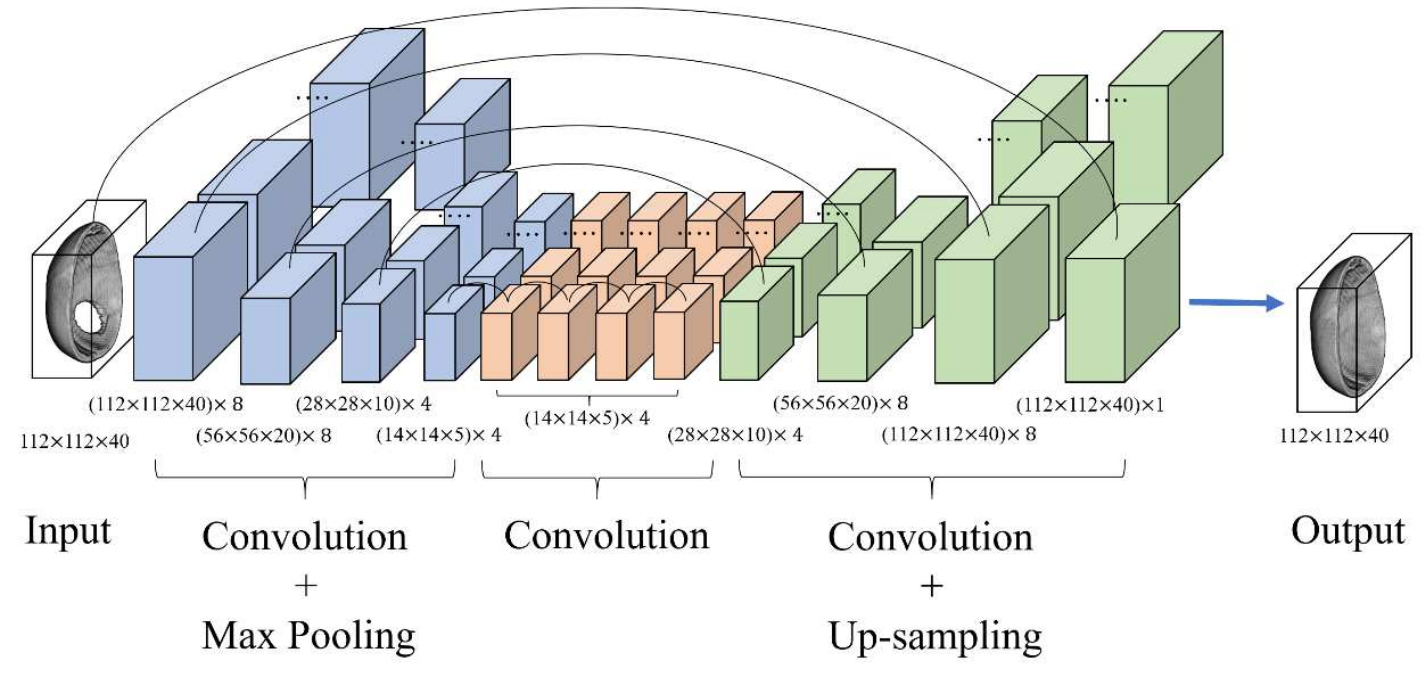

Figure 4. The proposed 12-layer deep learning model. 
Table 1. Architecture of the 3D inpainting network. "Channels" refers to the number of kernels for the corresponding layer.

\begin{tabular}{ccccc}
\hline Type & Kernel & Dilation & Stride & Channels \\
\hline 3D convolution & $3 \times 3 \times 3$ & 1 & $1 \times 1 \times 1$ & 8 \\
\hline 3D convolution & $3 \times 3 \times 3$ & 1 & $1 \times 1 \times 1$ & 8 \\
\hline 3D convolution & $3 \times 3 \times 3$ & 1 & $1 \times 1 \times 1$ & 4 \\
\hline 3D convolution & $3 \times 3 \times 3$ & 1 & $1 \times 1 \times 1$ & 4 \\
\hline dilated 3D convolution & $3 \times 3 \times 3$ & 2 & $1 \times 1 \times 1$ & 4 \\
dilated 3D convolution & $3 \times 3 \times 3$ & 4 & $1 \times 1 \times 1$ & 4 \\
dilated 3D convolution & $3 \times 3 \times 3$ & 8 & $1 \times 1 \times 1$ & 4 \\
dilated 3D convolution & $3 \times 3 \times 3$ & 16 & $1 \times 1 \times 1$ & 4 \\
\hline 3D deconvolution & $3 \times 3 \times 3$ & 1 & $1 \times 1 \times 1$ & 4 \\
\hline 3D deconvolution & $3 \times 3 \times 3$ & 1 & $1 \times 1 \times 1$ & 8 \\
\hline 3D deconvolution & $3 \times 3 \times 3$ & 1 & $1 \times 1 \times 1$ & 8 \\
\hline output & $3 \times 3 \times 3$ & 1 & $1 \times 1 \times 1$ & 1 \\
\hline
\end{tabular}

The data size of each $112 \times 112 \times 40$ skull model is $2 \mathrm{MB}$, and the 7,150 training sets amount to $14.35 \mathrm{~GB}$. To provide defective skull models for training, we randomly apply six types of 3D masks with equal probability: symmetrical ellipsoid, ellipsoid, mixed ellipsoid, cylinder, elliptical cylinder, and mixed elliptical cylinder, as shown in Fig. 5.

In training the network, a batch size of 10 models was applied, and we used Adadelta as the optimizer and Binary Cross entropy as the cost function. The time required for a training session of 1,200 epochs took 58.4 hours. The same settings were applied to train and evaluate our model. Once trained, a completion task takes only 8.6 seconds.

Details of the computational settings are provided in the Supplementary Material. 


\section{Creation of Simulated Defects on the Cranial Model}

\begin{tabular}{|c|c|c|}
\hline Type of 3D Mask & $\begin{array}{c}\text { Subtraction of Mask } \\
\text { from the Model }\end{array}$ & $\begin{array}{c}\text { Generated Defective } \\
\text { Model }\end{array}$ \\
\hline $\begin{array}{l}\text { Symmetrical } \\
\text { Ellipsoid }\end{array}$ & & \\
\hline Ellipsoid & & \\
\hline Mixed Ellipsoid & & \\
\hline Cylinder & & \\
\hline Elliptical Cylinde & & \\
\hline $\begin{array}{l}\text { Mixed Elliptical } \\
\text { cylinder }\end{array}$ & & \\
\hline
\end{tabular}

Figure 5. Various types of 3D masks are used to generate defects on the cranial model to train the proposed deep learning system. 


\section{References}

1. Alkhaibary, A. et al. Cranioplasty: A comprehensive review of the history, materials, surgical aspects, and complications. World Neurosurg. 139, 445-452 2020.

2. Sanan, A. \& Haines, S.J. Repairing holes in the head: A history of cranioplasty. Neurosurgery. 40, 588-603 1997.

3. Chen, X., Xu, L., Li, X. \& Egger, J. Computer-aided implant design for the restoration of cranial defects. Sci. Rep. 23, 4199 (2017).

4. Elharrouss, O., Almaadeed, N., Al-Maadeed, S. \& Akbari, Y. Image inpainting: A review. Neural Process. Lett. 51, 2007-2028 (2020).

5. Yan, Z., Li, X., Li, M., Zuo W. \& Shan, S. Shift-net: Image inpainting via deep feature rearrangement. The European Conf. on Comput. Vis. (ECCV). arXiv preprint arXiv: 1801.09392 (2018).

6. Ronneberger, O., Fischer, P. \& Brox, T. U-Net: Convolutional networks for biomedical image segmentation. Med. Image Comput. and Comput.-Assisted Interv. (MICCAI). Springer. LNCS 9351, 234-241 (2015).

7. Liao, L., Hu. R., Xiao, J. \& Wang, Z. Artist-net: decorating the inferred content with unified style for image inpainting. IEEE Access. 7,36921-36933 (2019).

8. Pathak, D. et al. Context encoders: feature learning by inpainting. arXiv preprint arXiv:1604.07379 (2016).

9. Hinton, G.E. \& Salakhutdinov, R.R. Reducing the dimensionality of data with neural networks. Science. 313, 504-507 (2006).

10. Vincent, P., Larochelle, H., Bengio, Y. \& Manzagol, P.A. Extracting and composing robust features with denoising autoencoders. In Proc. of the 25th Int. Conf. on Machine Learning (ICML '08), 1096-1103 (2008).

11. Baldi, P. Autoencoders, unsupervised learning, and deep architectures. ICML Workshop on Unsupervised and Transfer Learning. 27, 37-49 (2012).

12. Goodfellow, I.L. et al. Generative adversarial networks. Int. Conf. on Neural Inf. Process. Syst. (NIPS 2014). 2672-2680 (2014).

13. Iizuka, S., Edgar, S.-S., and Ishikawa, H. Globally and locally consistent image completion. ACM Trans. Graph. 36, 107. https://doi.org/10.1145/3072959.3073659 (2017).

14. Wang, Q., Fan, H., Zhu, L. \& Tang, Y. Deeply supervised face completion with multi-context generative adversarial network. IEEE Signal Process. Lett. 26, 400404 (2019).

15. Jiang, Y., Xu, J., Yang, B., Xu, J. \& Zhu, J. Image inpainting based on generative adversarial networks. IEEE Access. 8, 22884-22892 (2020).

16. Han, X., et al. High-resolution shape completion using deep neural networks for global structure and local geometry inference. In Proceedings of IEEE Int. Conf. Comput. Vis. (ICCV). https://doi.org/10.1109/ICCV.2017.19 (2017).

17. Wang, W., et al. Shape inpainting using 3D generative adversarial network and recurrent convolutional networks. In Proceedings of IEEE Int. Conf. Comput. Vis. (ICCV). https://doi.org/10.1109/ICCV.2017.252 (2017).

18. Dai, A., et al. Shape completion using 3D-encoder-predictor CNNs and shape synthesis. In Proc. of IEEE Int. Conf. Comput. Vis. (ICCV). https://doi.org/10.1109/CVPR.2017.693 (2017).

19. Wang, X., Xu, D. \& Gu, F. 3D model inpainting based on 3D deep convolutional 
generative adversarial network. IEEE Access. 8, 170355-170363 (2020).

20. Morais, A., Egger, J. \& Alves, V. Automated computer-aided design of cranial implants using a deep volumetric convolutional denoising autoencoder. World Conf. on Inf. Syst. and Tech., 151-160 (2019).

21. Li, J. et al. A baseline approach for AutoImplant: the MICCAI 2020 Cranial Implant Design Challenge. arXiv preprint arXiv:2006.12449 (2020).

22. Shi, H. \& Chen, X. Cranial implant design through multiaxial slice inpainting using deep learning. AutoImplant 2020. Springer. LNCS 12439, 28-36 (2020).

23. Matzkin, F. et al. Self-supervised skull reconstruction in brain CT Images with decompressive craniectomy. Med. Image. Comput. Comput. Assist. Interv. Springer. LNCS 12262, 390-399 (2020).

24. Matzkin, F., Newcombe, V., Glocker, B. \& Ferrante, E. Cranial implant design via virtual craniectomy with shape priors. arXiv preprint arXiv:2009.13704 [eess.IV] (2020).

25. Seeram, E. Computed Tomography: Physical Principles, Clinical Applications, and Quality Control. Elsevier Health Sciences (2015).

26. Shorten, C. \& Khoshgoftaar, T.M. A survey on image data augmentation for deep learning. J. of Big Data. 6, 60 (2019).

\section{Acknowledgements}

This work was supported by Grants from the Ministry of Science and Technology, Taiwan, under grant numbers MOST 108-2221-E-182-061 and MOST 109-2221-E182-025; and Chang Gung Memorial Hospital, Taiwan, under grant numbers CORPD2J0041, CORPD2J0042, CORPD2H0011 and CORPD2H0012.

\section{Author information}

Affiliations

Department of Mechanical Engineering, Chang Gung University, Taoyuan, Taiwan

Yao-Hung Yang \& Yau-Zen Chang

Department of Neurosurgery, Chang Gung Memorial Hospital, Taoyuan, Taiwan

Chieh-Tsai Wu \& Yau-Zen Chang

\section{Author Contributions}

Dr. C.T.W. collected and screened the dataset and performed the clinical treatments. Y.H.Y. preprocessed the dataset and built and trained the deep learning system under the supervision of Dr. C.T.W. and the guidance of Prof. Y.Z.C. Prof. Y.Z.C. wrote the Manual script and all the other two authors reviewed it. Prof. Y.Z.C. is the one responsible for submission and is the corresponding author. 


\section{Ethics declarations}

Competing interests

The authors declare no competing interests.

\section{Additional information}

Supplementary information is available for this paper.

Correspondence and requests for materials should be addressed to Y.Z. 

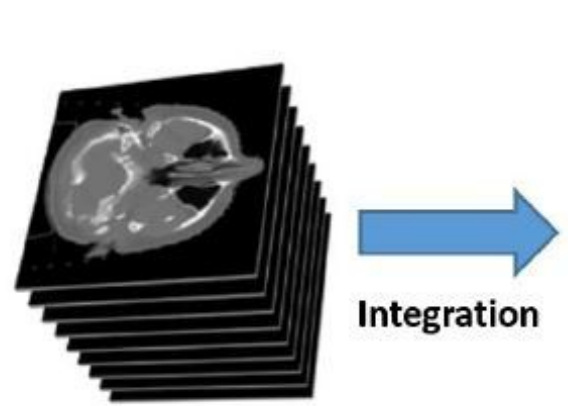

CT-Scanned Image Set

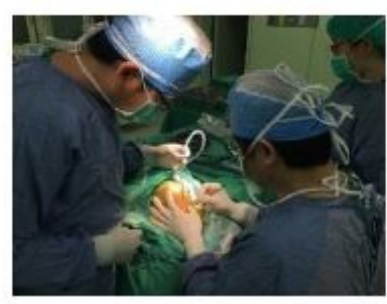

Repair Surgery

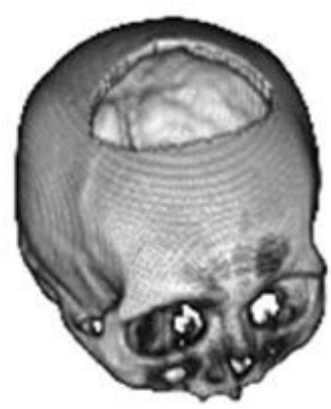

Original 3D Model

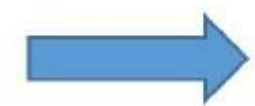

The Proposed 3D Deep Learning System

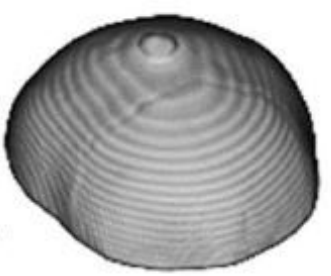

Completed 3D Model
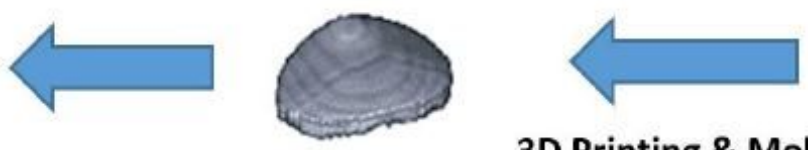

3D Printing \& Molding

Bone Cement Implant

Figure 1

Cranial reconstruction surgery flowchart. 


\section{Inpainting Defective Cranial Models by the Proposed Scheme}

\begin{tabular}{|c|c|c|c|c|}
\hline Defect Type & Cylinder & Irregular Cylinder \\
\hline $\begin{array}{c}\text { Defective } \\
\text { Skull } \\
\text { (Top View) }\end{array}$ \\
\hline $\begin{array}{c}\text { Completed } \\
\text { Skull } \\
\text { (Top View) }\end{array}$ \\
\hline $\begin{array}{c}\text { Defective } \\
\text { Skull } \\
\text { (Isometric } \\
\text { View) }\end{array}$ \\
\hline $\begin{array}{c}\text { Completed } \\
\text { Skull } \\
\text { (Isometric } \\
\text { View) }\end{array}$ \\
\hline $\begin{array}{c}\text { Ground- } \\
\text { Truth } \\
\text { Implant }\end{array}$ \\
\hline $\begin{array}{c}\text { Generated } \\
\text { Implant }\end{array}$ \\
\hline $\begin{array}{c}\text { Volumetric } \\
\text { Error Rate }\end{array}$
\end{tabular}

\section{Figure 2}

Automatic generation of implants for defective cranial models by the proposed deep learning system. The defects are made by Boolean subtraction of 4 types of 3D masks from an intact skull model. 


\section{Surgical Treatment of a Cranial Defect}

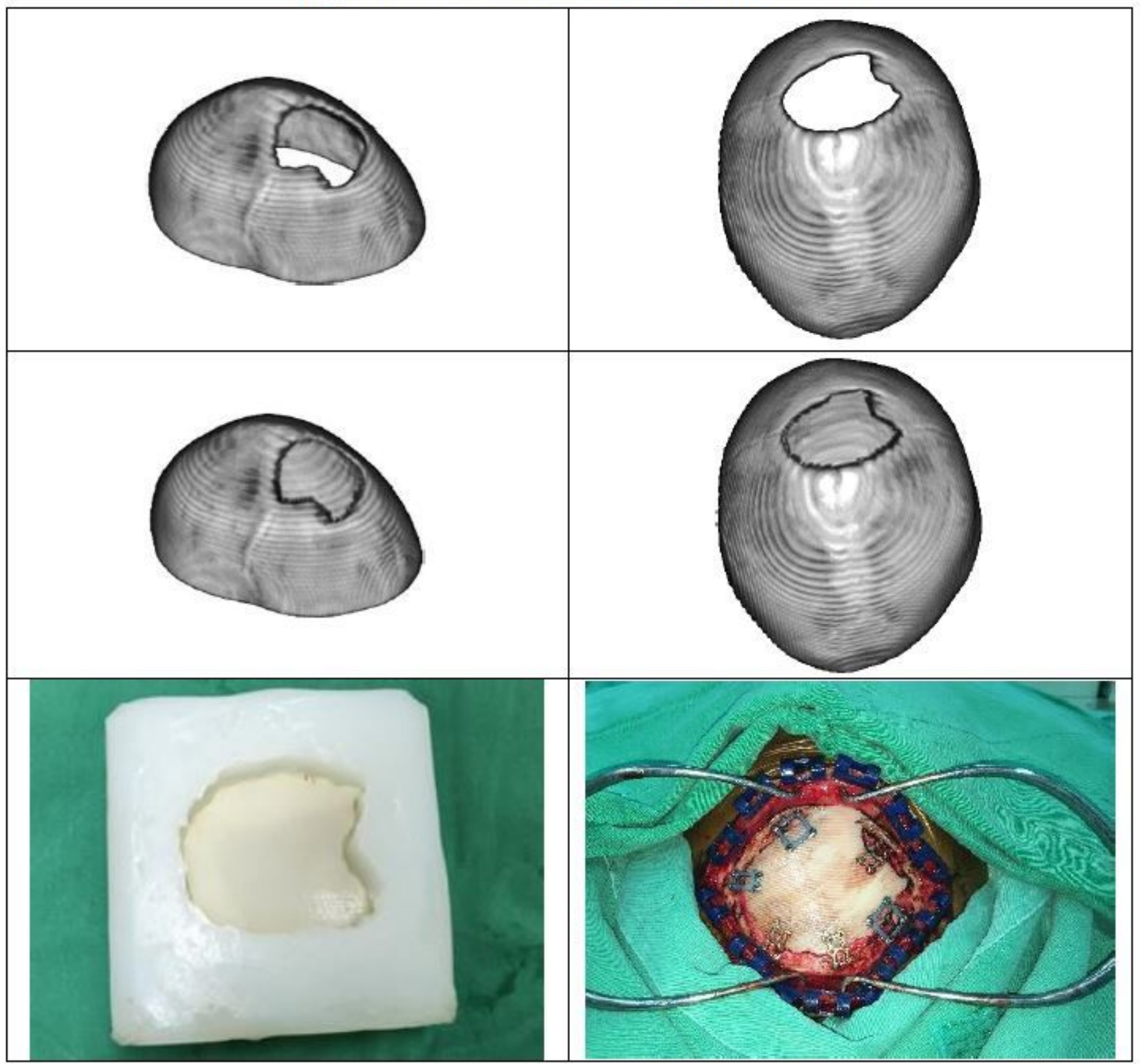

\section{Figure 3}

Clinical cranial reconstruction. The first row shows the isometric and top views of the original cranial model. The second row shows the isometric and top views of the repaired cranial model using the implant created by the proposed deep learning system. The last row shows the bone-cement implant molded with silicone rubber (left photo), and a scene of the surgery when the implant was fixed to the skull (right photo). 


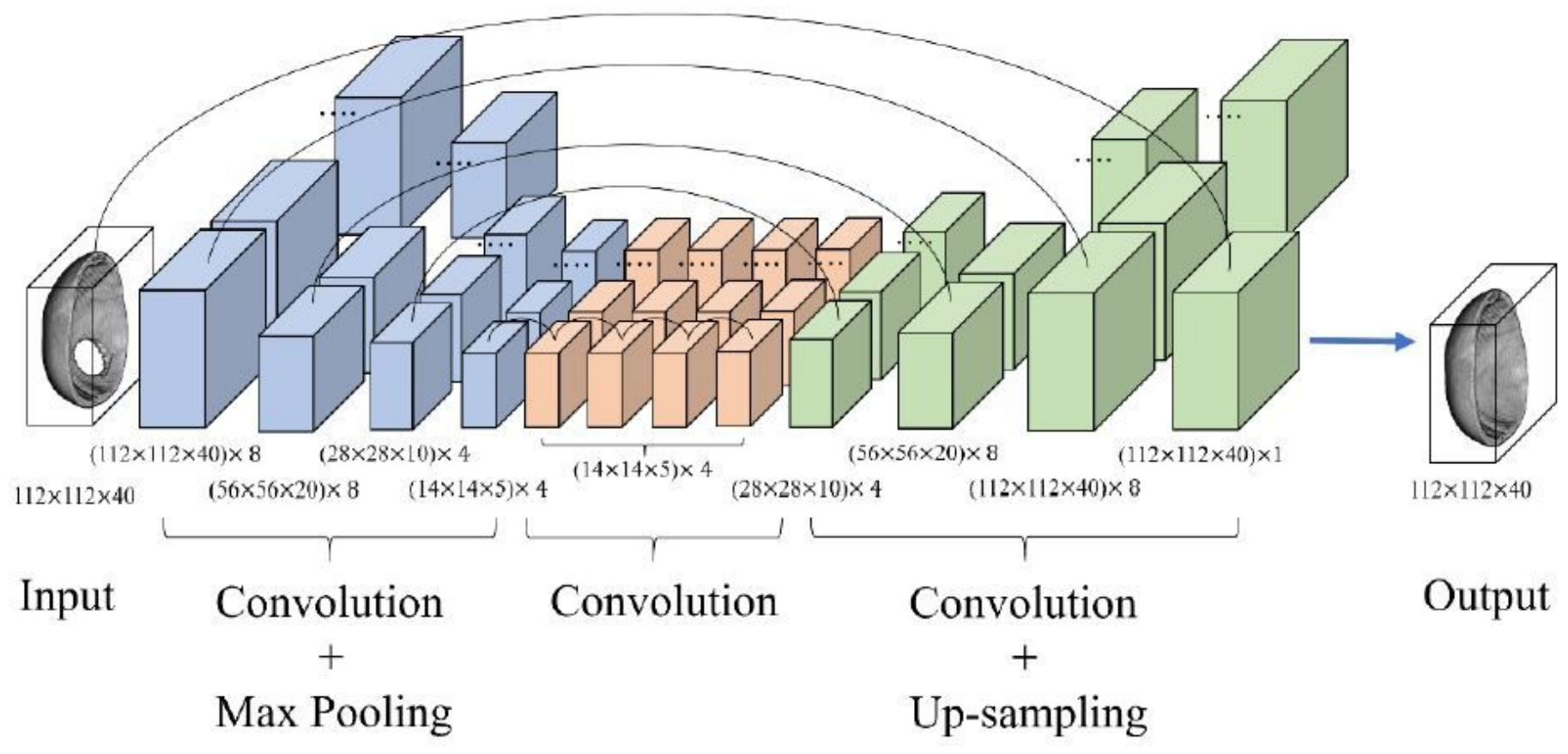

Figure 4

The proposed 12-layer deep learning model. 
Creation of Simulated Defects on the Cranial Model

\begin{tabular}{|c|c|c|}
\hline $\begin{array}{c}\text { Type of 3D Mask } \\
\text { Ellipsoid }\end{array}$ & $\begin{array}{c}\text { Subtraction of Mask } \\
\text { from the Model }\end{array}$ & $\begin{array}{c}\text { Generated Defective } \\
\text { Model }\end{array}$ \\
\hline Ellipsoid & \\
\hline Mixed Ellipsoid & \\
\hline Clliptical Cylinder
\end{tabular}

\section{Figure 5}

Various types of 3D masks are used to generate defects on the cranial model to train the proposed deep learning system.

\section{Supplementary Files}

This is a list of supplementary files associated with this preprint. Click to download.

- Supplementarymaterial.pdf 